Check for updates

Cite this: RSC Adv., 2019, 9, 27199

\title{
Highly monodisperse zwitterion functionalized non-spherical polymer particles with tunable iridescence $\uparrow$
}

\author{
Vivek Arjunan Vasantha, (D) *a Wendy Rusli, ${ }^{\text {a }}$ Chen Junhui, ${ }^{a}$ Zhao Wenguang, ${ }^{a}$ \\ Kandammathe Valiyaveedu Sreekanth, ${ }^{\text {bc }}$ Ranjan Singh ${ }^{\text {bc }}$ \\ and Anbanandam Parthiban (D) *a
}

\begin{abstract}
A facile and simple synthetic route towards functionalized non-spherical polymer particles (NSP) with tunable morphologies and iridescence is presented. Monodisperse particles with unique zwitterionic functionality were synthesized via emulsifier-free emulsion polymerization in a single step process. The sulfobetaine comonomer was utilized to induce phase separation in the course of polymerization to achieve anisotropic NSP with controlled morphologies such as quasi-spherical with protruding structures like bulge, eye-ball, and snowman-like nanostructures. Both SEM and TEM analyses revealed anisotropic particles, and phase-separated protrusion morphology with a small increase in aspect ratio. By taking advantage of the monodisperse, colloidally stable NSPs, template free photonic crystal arrays were fabricated through a bottom-up approach. The particles readily self-assemble and exhibit a photonic bandgap with vivid structural colors that arise from ordered structures of different morphologies. Additionally, the salt-responsive photonic crystals also possess tunable color-changing characteristics.
\end{abstract}

Received 8th July 2019

Accepted 14th August 2019

DOI: 10.1039/c9ra05162g

rsc.li/rsc-advances studies on single or multi-component polymer colloids have been limited to spherical particles. ${ }^{18-20}$ Moreover, the stability of colloids depends on the layer structure (deposition pattern, spacing, and packing arrangement), composition as well as particle type and size which could limit their assembly behavior and applications. ${ }^{21-23}$ Extending the colloidal particles from conventional shape to anisotropic dimensions with control over their spatial orientation by influencing geometries, chemical composition, void and deposition pattern have been explored in physical, chemical and material science. ${ }^{24}$

Colloidal non-spherical particles (NSP) constitute a novel class of colloid with the patchy, bulge or dumbbell shape and Janus architecture. These slightly anisotropic structures and their properties have drawn extensive attention recently. In contrast to spherical particles, NSPs provide an alternative building block that can spontaneously organize to form complex structures as new bio and engineering functional materials. ${ }^{25}$ Recently, NSPs with distinct properties have been considered as advanced materials for controlling photonic bandgap. ${ }^{26,27}$ Monodisperse colloidal NSPs with precise control over size, shape, chemical composition, and tunable functionality are highly desired for various applications. ${ }^{26,28}$ In the past decade, numerous strategies have been developed to design and prepare non-spherical colloids with a wide range of particles of different shapes. ${ }^{29,30}$ However, effectively producing these particles with uniform non-spherical shape along with a chosen surface chemistry that is suitable for the fabrication of PC to achieve structural-color, is still challenging. Additionally, the 
process of making monodisperse NSPs is usually accompanied with the formation of undesired morphologies.

In order to achieve the aforementioned control, seeded emulsion copolymerization offers potential advantages over other synthetic methods for producing NSPs in large quantities. $^{30-33}$ It enables the generation of nanoscale to microscale featured structures by a single or multi-step process. ${ }^{34}$ However, there are challenges for making monodisperse NSPs due to the problems associated with the choice of comonomers, particle agglomeration and stability, controlling the size, uniformity, and scale-up. We hereby report a simple and versatile approach to prepare monodisperse NSP in high yield using a sulfobetaine comonomer. The evaporation-driven self-assembly at the airwater interface has been used to fabricate functional PC array which facilitates Bragg diffraction, thereby showing tunable iridescent behavior of polymer opal. Also, the photonic properties of the film can be easily controlled by varying the anisotropy of NSPs or by simply changing the salt concentration.

\section{Results and discussion}

\section{Zwitterion functionalized microspheres via emulsifier (i.e. surfactant)-free emulsion polymerization}

The emulsifier or surfactant-free $a b$ initio polymerization process that was employed to prepare zwitterion functionalized polymer colloids is illustrated in Fig. 1a. In the first stage of preparation, typical emulsion polymerization by persulfate initiation leads to the formation of polystyrene (PS) seed particles. The zwitterionic comonomer (sulfobetaine, SB) was added subsequently. The adsorption of SB comonomer on PS seed helps to control the size and stabilization of the seed. SB comonomer also promotes the formation of the monomer droplets of styrene which in turn helps to swell the seed as well as act as a reservoir of monomer for propagation. SB comonomer with a hydrophilic-lipophilic balance (HLB) of 11.8 plays a vital role as a reactive emulsifier. This nature of SB helps to stabilize as well as to capture the aggregated seed and also simultaneously copolymerize to form the charged functional surface. In the case of PS/PSB 1 (Table 1, entry 1) an increase in average particle size was observed during the course of polymerization from $170 \mathrm{~nm}$ to $400 \mathrm{~nm}$ after introducing the comonomer SB. The particles were highly monodisperse. Fig. 1b shows the size and morphology of PS/PSB 1 colloids as studied using DLS and SEM. The volume-average hydrodynamic diameter $\left(D_{\mathrm{v}}\right)$ of $455 \pm 10 \mathrm{~nm}$ was noticed with a monomodal particle size distribution of 0.02. The SEM and TEM images confirmed that the polymerization process produced highly monodisperse functional polymer microspheres with the size of $367 \pm 18 \mathrm{~nm}$ and coefficient of variation (CV) of 1.5\% (Fig. S1†). From these results, it can be seen that PS particles are well functionalized by sulfobetaine which provides the electrostaticsteric stability of the polymer latex particles, improve adhesion through surface hydrophilicity and offer unique functional sites for further modification. Besides, this process leads to the facile formation of colloidal PC array with high uniformity and smooth surface via self-assembly of PS/PSB 1 resulting in

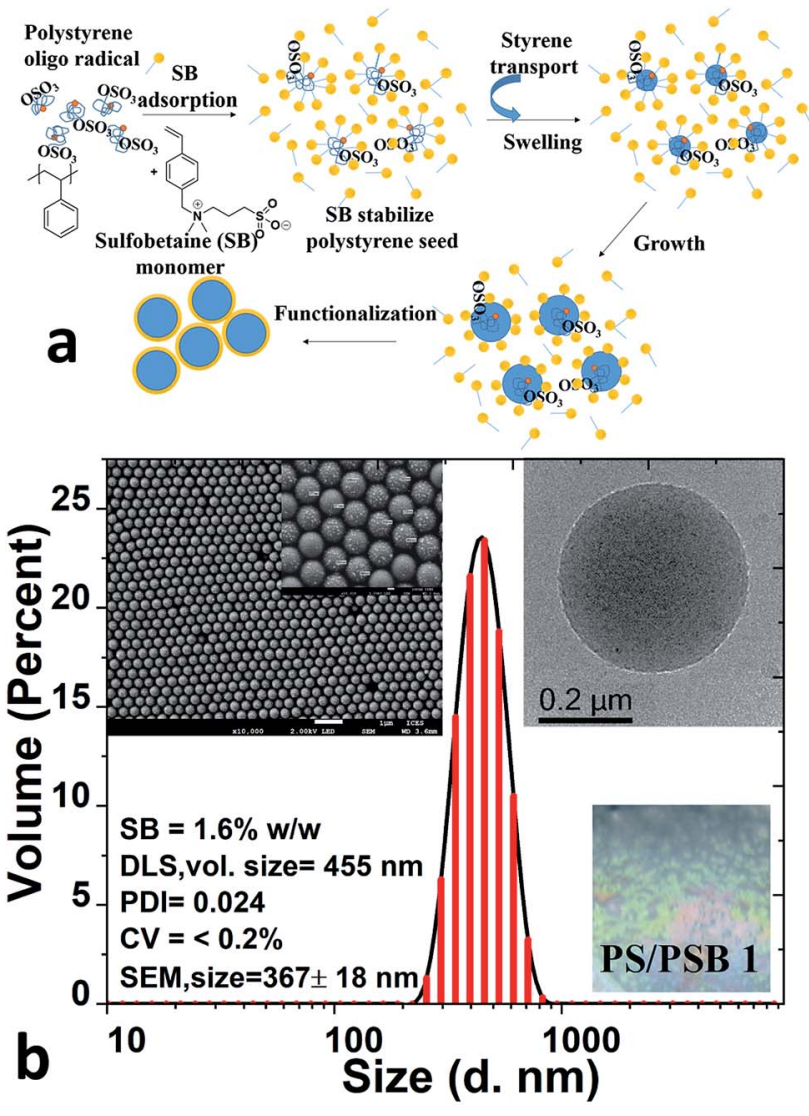

Fig. 1 (a) Schematic illustration of the steps involved in the preparation of NSP, PS/PSB; (b) the monomodal size distribution of PS/PSB 1 as determined by DLS and SEM (inset: top left; scale bar $1 \mu \mathrm{m}$ and inset of SEM $100 \mathrm{~nm}$ ); diameter $=373 \pm 7 \mathrm{~nm}$ and cryo-TEM (inset: top right; scale bar $200 \mathrm{~nm}$ ). (Inset: bottom right - photo image of polymer opal PC array self-assembled on a silica substrate at room temperature).

polymer opal (Fig. 1b inset, bottom right). The self-assembly of colloidal PS/PSB as a PC were detailed in a latter section.

\section{Self-assembly induced phase separation - influence of sulfobetaine comonomer}

The emulsifier-free $a b$ initio emulsion polymerization yielding different nanostructures is a result of self-assembly induced by phase separation in contrast to polymerization induced selfassembly (PISA) process. PISA produces a wide range of nanostructures such as spherical and cylindrical micelles and vesicles. $^{35,36}$ The process reported here is fundamentally different from PISA in many respects. In the case of PISA, a strongly hydrophilic or water-soluble monomer is polymerized to obtain a hydrophilic block which is followed by the polymerization of the hydrophobic monomer to form amphiphilic block copolymers. The nanostructures mentioned above are formed from the self-assembly of these block copolymers. The process reported here involves the formation of hydrophobic seed, and the hydrophilic monomer is introduced later. The process also does not comprise any chain transfer agent. Significantly, the concentration of hydrophilic units in the final polymer is far lower than that of those produced by PISA. 
Table 1 Emulsion polymerization of styrene and SB using KPS as initiator and characteristics of PS/PSB particles ${ }^{a}$

\begin{tabular}{|c|c|c|c|c|c|c|c|}
\hline Entry & Polymer code & Sty : SB : initiator : water $(\% \mathrm{w} / \mathrm{w})$ & ${ }^{b} \mathrm{SB}(\% \mathrm{w} / \mathrm{w})$ & ${ }^{c}$ Conversion $(\%)$ & $\begin{array}{l}d_{z \text {-Average diameter, }} \\
\text { DLS (nm) }\end{array}$ & ${ }^{d}$ PDI, DLS & ${ }^{e}$ Aspect ratio, SEM \\
\hline 1 & PS/PSB 1 & $7.88: 0.12: 0.07: 91.92$ & 1.5 & 86.8 & $423 \pm 6$ & 0.02 & - \\
\hline 2 & PS/PSB 2 & $7.61: 0.24: 0.07: 92.08$ & 3.1 & 84.2 & $320 \pm 4$ & 0.07 & $1.09 \pm 0.05$ \\
\hline 3 & PS/PSB 3 & $7.85: 0.49: 0.07: 91.58$ & 6.1 & 85.3 & $251 \pm 2$ & 0.03 & $1.12 \pm 0.05$ \\
\hline 4 & PS/PSB 4 & $7.57: 0.72: 0.07: 91.64$ & 8.6 & 91.2 & $264 \pm 3$ & 0.02 & $1.13 \pm 0.05$ \\
\hline 5 & PS/PSB 5 & $7.56: 0.83: 0.07: 91.54$ & 9.9 & 91.9 & $296 \pm 2$ & 0.01 & $1.15 \pm 0.06$ \\
\hline
\end{tabular}

Polysulfobetaines (PSB) exhibit phase separation because of the strong interaction between polymer chains in aqueous medium. ${ }^{37-39}$ The strong electrostatic and hydrophobic interactions prevailing in SB comonomer could be utilized to induce phase separation during emulsion polymerization, as illustrated in Fig. 2a. Here we systematically varied the content of SB in the range of 1.5 to $10 \%(\mathrm{w} / \mathrm{w})$ to styrene. The concentration of styrene and initiator were kept constant to obtain functional PS/ PSB particles. The shape uniformity and diameter of PS/PSB colloids were determined by DLS and SEM analyses. As shown in Fig. 2b, S2, $\dagger$ and Table 1, the hydrodynamic particle diameter decreased and then increased with increasing SB content. In all cases, the particles remained monodisperse. When the concentration of SB was $<3 \% \mathrm{w} / \mathrm{w}$, sphere and quasi-sphere morphology were observed with sizes of $455 \pm 10 \mathrm{~nm}\left(D_{\mathrm{v}}\right)$ and $365 \pm 7 \mathrm{~nm}\left(D_{\mathrm{v}}\right)$, respectively. The corresponding SEM of PS/PSB 1 and PS/PSB 2 and DLS size distribution are presented in Fig. 1 and 2c (top left), and Fig. S1 and S2a. $\dagger$

More importantly, increasing SB content from $2 \%$ to $6 \% \mathrm{w} / \mathrm{w}$ triggers an increase in the bulge and show different nanostructures with a slight increase in the aspect ratio from 1 to 1.12. Fig. 2c shows that the SEM images of PS/PSB particles mostly consist of uniform non-spherical morphologies such as bulge, eye-ball, and snowman-like morphologies. The particle size $\left(D_{\mathrm{v}}\right)$ is about $268 \pm 6 \mathrm{~nm}$ (PS/PSB 3), $278 \pm 3 \mathrm{~nm}$ (PS/PSB 4), and $296 \pm 2 \mathrm{~nm}$ (PS/PSB 5), respectively. The particle size distribution and the aspect ratio (length/diameter) of PS/PSB particles are below 0.1 and less than 1.2 respectively. However, above $6 \% \mathrm{w} / \mathrm{w}$ of SB, the size of the bulge (PS/PSB) moderately increased representing snowman-like nanostructure (Fig. 2c, bottom left). The aspect ratio of protruded PS/ PSB 4 and PS/PSB 5 were higher, with an increase in $z$-average diameter of about 20-50 nm (Fig. 2b).

Clearly, these polymer particles have distorted, or phaseseparated nanostructures with structural integrity, low aspect ratio $(<1.2)$ and uniform size distribution as revealed by both SEM and TEM analyses (Fig. S2 $\dagger$ ). The protrusion noticed in the nanostructure could either be a result of hydrophobic, van der Waal's interaction between non-ionic part of SB comonomer and the PS core particle or due to the phase separation induced by the substantial difference in polarity between comonomers or a combination of both. ${ }^{40-42}$ The protruded nanostructure is further confirmed by the cryo-TEM analysis. Cryo-TEM and TEM analyses show the presence of slightly anisotropic NSP (Fig. 2b and d).

NSP colloids and NSPs were further characterized by zeta potential, elemental analysis, XPS, FTIR, and TGA (Table S2 and Fig. S3-S5 $\dagger$ ) techniques. The corresponding volume fraction, particle number, and the surface areas of PS/PSB particles are summarized in Table S1. $\dagger$ It is also possible to obtain monodisperse sphere, quasi-sphere and protruded structures such as bulge, eye-ball, and snowman-like morphologies in multi-gram scale with a high yield just by controlling the sulfobetaine comonomer content in a one-pot polymerization. Therefore, the emulsion polymerization using SB comonomer opens up possibilities to prepare functional anisotropic colloids with tunable morphologies.

\section{Colloidal stability of non-spherical PS/PSB particles}

The facile and straightforward synthesis of monodisperse PS/ PSB colloids via surfactant free emulsion polymerization was possible due to the electrostatic repulsion between particles. The colloidal stability of NSP of PS/PSB was evaluated by using DLS and zeta potential measurements (Fig. 3). The electrophoretic mobility of PS/PSB is about -4.5 to $-3.5 \mu \mathrm{m} \mathrm{cm} \mathrm{V} \mathrm{s}^{-1}$ in $1 \mathrm{mM} \mathrm{NaCl}$. Fig. 3a shows the zeta potential of PS/PSB in $1 \mathrm{mM}$ $\mathrm{NaCl}$. All the particles presented a strongly negative charge within the range of -57 to $-45 \mathrm{mV}$. In DI water, the zeta potential of PS/PSB was in the range of $-49 \mathrm{mV}$ to $-45 \mathrm{mV}$.

The negative potential indicates that the surface of the particles is negatively charged as expected from an ionic charge of sulfonate of zwitterion and sulfate from KPS initiator on the surface. Despite the charge neutrality of zwitterionic surface, the zeta potential of PS/SPBs presented a strong negative charge, which indicated electrostatic stabilization of the dispersion is very significant. ${ }^{43-45}$ Moreover, sulfobetaine functionalized particles allowed better electrostatic repulsion between charged particles and facilitated the stabilization in solution without the addition of any additives. However, upon increasing the SB content in PS/PSB 3 and PS/PSB 4, the mobility lowered thereby, showing that the electrostatic repulsion is lower than that of PS/PSB 1 and 2. Evidently, the NSP of 
a

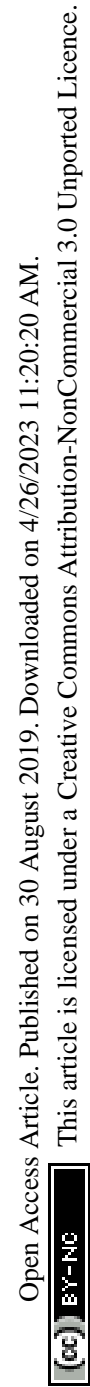

d
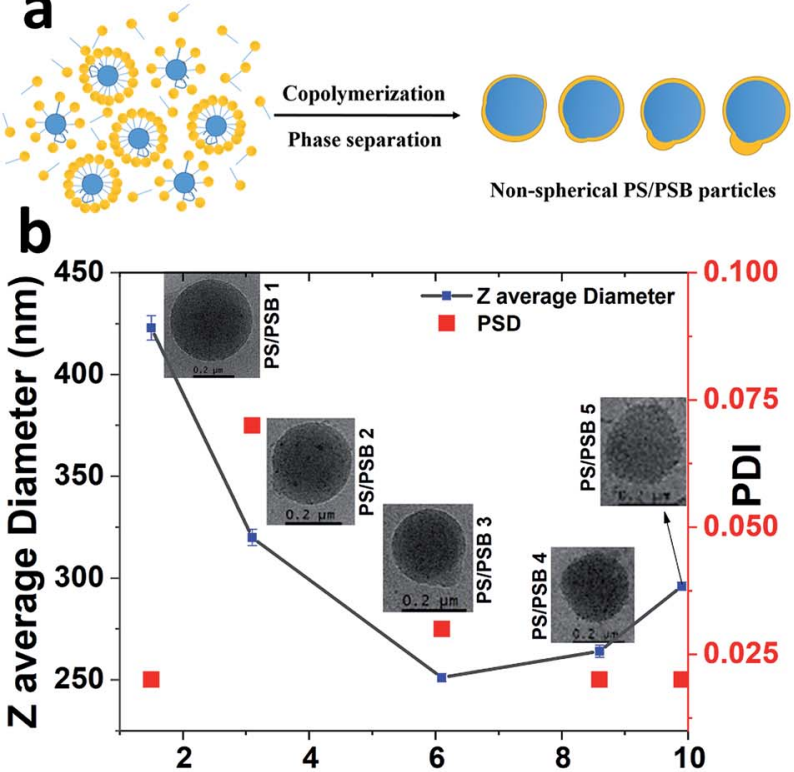

C Comonomer content (wt \%)
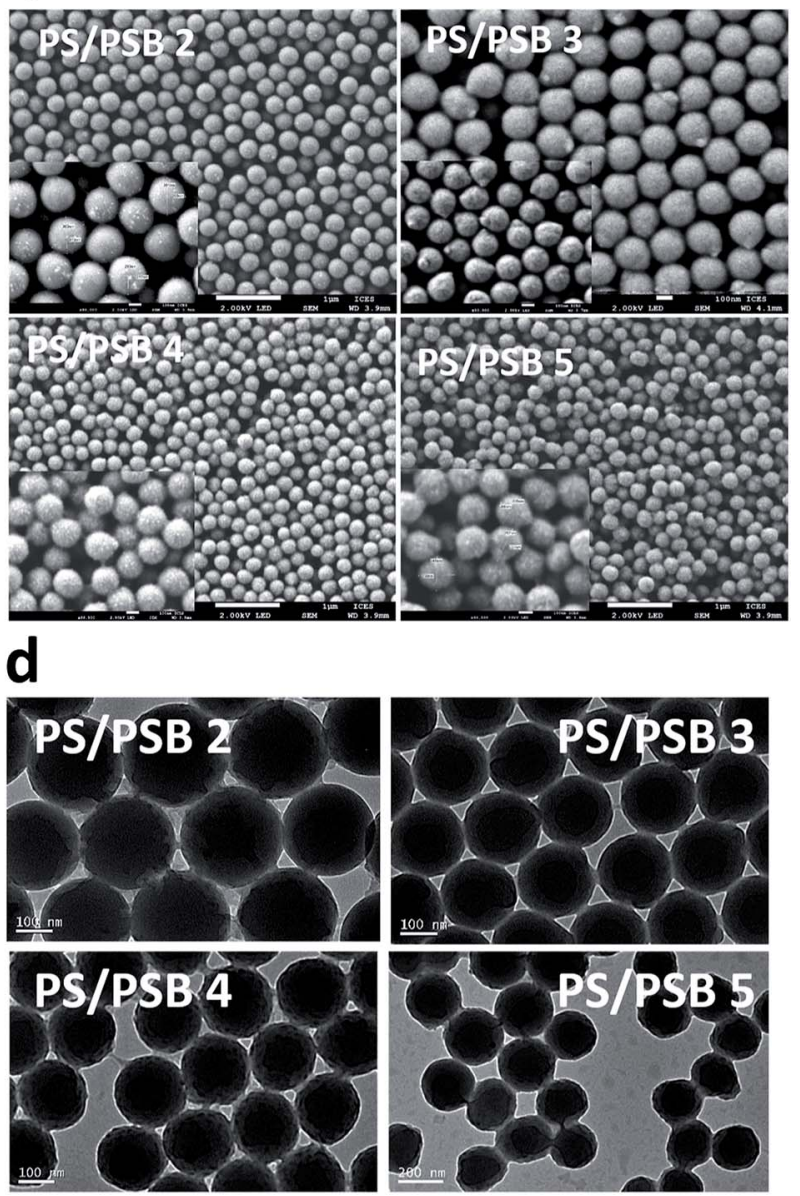

Fig. 2 (a) Schematic representation of the preparation of phase separated non-spherical PS/PSB colloids; (b) the effect of concentration of sulfobetaine comonomer on particle diameter (inset: cryo-TEM of PS/PSB particles; scale bar = $200 \mathrm{~nm}$ ); (c) SEM images of dry PS/PSB particles; PS/ PSB 2 (top left); PS/PSB 3 (top right); PS/PSB 4 (bottom left); PS/PSB 5 (bottom right) (inset: magnified view of NSP. Scale bar $1 \mu \mathrm{m}$ and inset 100 $\mathrm{nm}$ ); (d) TEM of dry PS/PSB particles; PS/PSB 2 (top left); PS/PSB 3 (top right); PS/PSB 4 (bottom left); PS/PSB 5 (bottom right) (scale bar $100 \mathrm{~nm}$ ).
PS/PSB can be stabilized in electrolytes such as brine, thereby showing halophilic (salt-loving) character $^{37,38,44}$ of sulfobetaine based surface (Fig. 3b). The result further confirmed the role of the sulfobetaine functionalized non-spherical colloids as an electrostatic stabilizer as well as a steric stabilizer. Both of these factors might be responsible for enhancing the colloidal stabilization in a complex mixture like high brine solution. The NSP colloids are very stable as no aggregation or coagulation was observed over two months. Thus, sulfobetaine functionality not only acts as a novel stabilizer for the polystyrene core but also imparts other key characteristics like salt responsiveness.

\section{Self-assembly of non-spherical PS/PSB particles}

Fig. 4 and S6† show typical morphologies of self-assembled functional PC arrays upon evaporation of water from colloidal NSP dispersion of PS/PSB cast on a silica substrate without the aid of a template. SEM images show a highly ordered selfassembled stacking of NSPs with a closely packed arrangement (Fig. 4(iv)). The fabricated NSPs are arranged hexagonally with few defects (Fig. 4 and S6†). The protruding morphology of NSPs helps to decrease the void between adjacent particles by

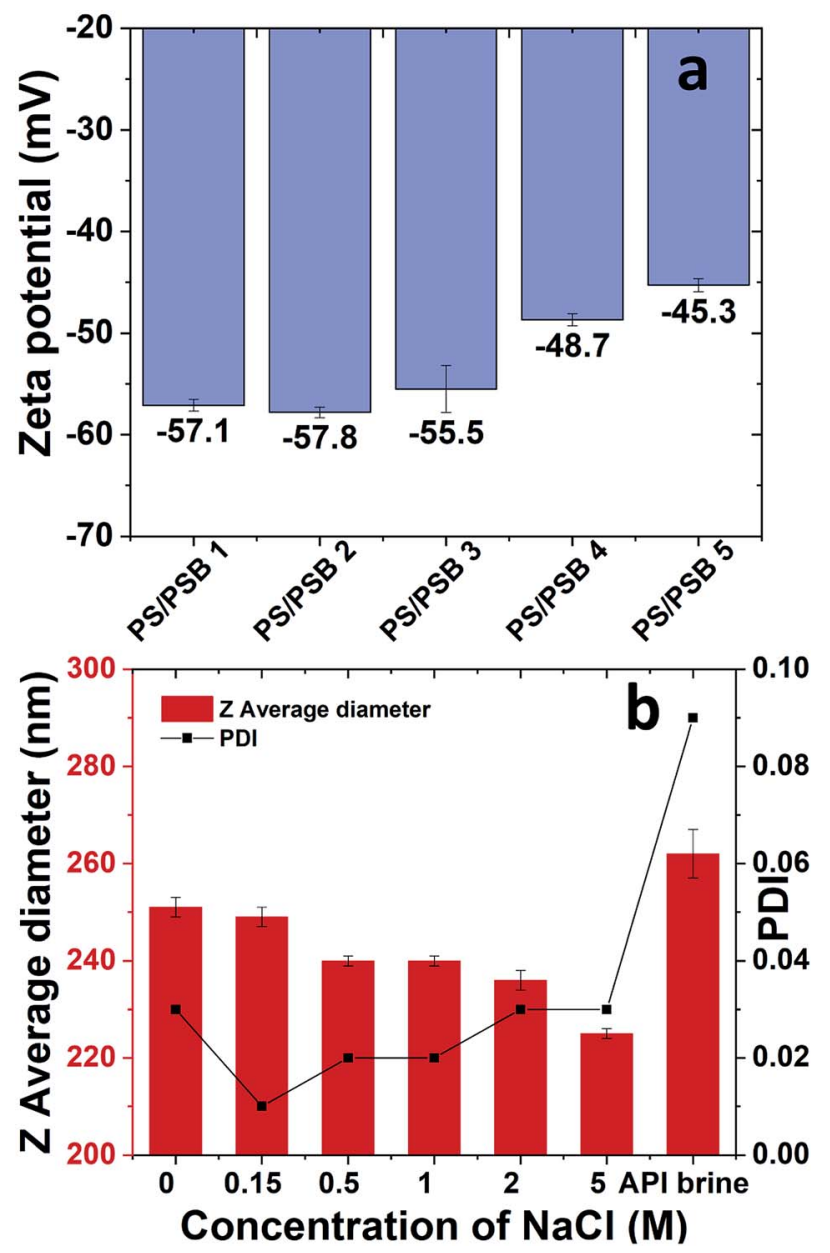

Fig. 3 (a) Zeta potential of PS/PSB in $1 \mathrm{mM} \mathrm{NaCl}$ at $25^{\circ} \mathrm{C}$; (b) the colloidal stabilization of PS/PSB 3 in different salt concentration and API (American Petroleum Institute) brine $\left(8 \% \mathrm{NaCl}\right.$ and $\left.2 \% \mathrm{CaCl}_{2}\right)$. 

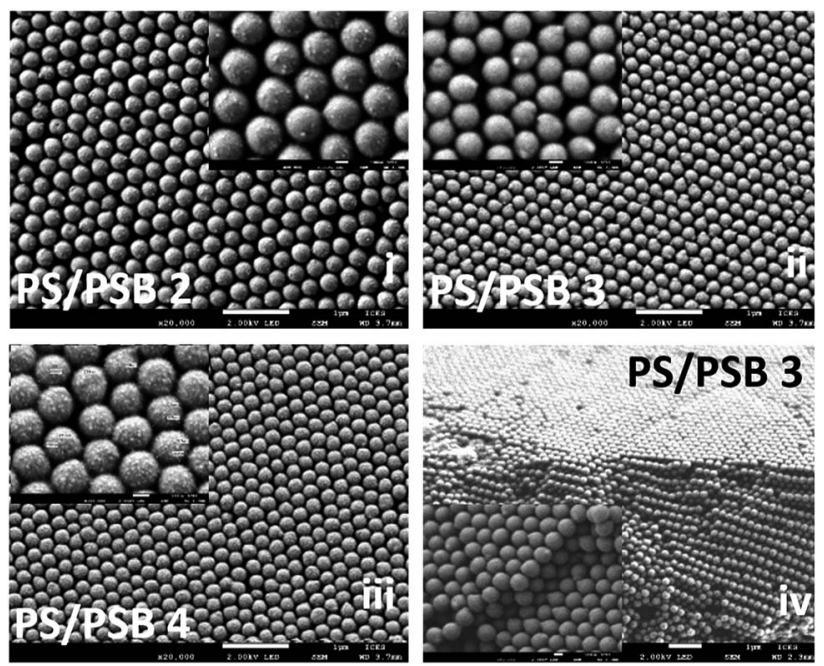

Fig. 4 SEM images of top view of self-assembled NSP colloidal PC films of (i) PS/PSB 2, (ii) PS/PSB 3 (iii) PSB 4 with hexagonally packed arrangement and (iv) the cross-section of highly ordered PS/PSB 3 colloidal PC array (inset: magnified view of a PC array; scale bar $=1 \mu \mathrm{m}$ and inset $=100 \mathrm{~nm}$ )

filling the vacant space. However, some voids still exist or are partially filled due to the change in orientation of protrusion. The layered structure of PS/PSB 2, 3, and 4 are entirely different from each other (Fig. 4a(i)-(iii)). This shows that the slight change in anisotropy of NSP could significantly influence the colloidal self-assembled nanostructure. We believe that the patchy protruded NSP nanostructure offers alternative packing arrangements that are not possible with spherical particles.

PS/PSB 5 with the highest SB content has a significant influence on the packing of particles. As shown in Fig. S7, $\uparrow$ the interparticle distance substantially increases with the sulfobetaine content. This is most likely due to the increased hydrophilicity of the surface, which enhances water uptake by the particles. As a result, after drying, the interparticle distance increased after shrinking of particles. The NSPs are aligned in a different direction with poor (non-) contact between adjacent particles. Ultimately the electrostatic repulsion and steric constraint of zwitterionic moieties on the surface influence the packing arrangement and orientation of particles. Thus, by altering the composition of $\mathrm{SB}$, it is possible to control the particle size, spacing between particles and therefore the packing arrangement which could in turn help to tune the optical properties.

\section{Tunable iridescence of PS/PSB particles}

Importantly, these ordered functional NSPs exhibit a brilliant structural holographic iridescence. The reflectance measurement of the functional NSP at normal and oblique incidence was measured to prove the existence of photonic bandgap. Fig. 5a shows the experimentally obtained reflection spectra of the samples PS/PSB 1 to 5 at normal incidence. The spectra reveal Bragg reflection peaks at $\lambda_{\max }=527,660,529,534$ and $564 \mathrm{~nm}$ respectively. The corresponding photograph of the samples obtained at the normal incidence is shown in the inset of Fig. 5a. It is evident that the reflection peak wavelength of each sample almost matches with the color of the corresponding sample, which is a clear sign of photonic bandgap. The angular reflection spectra of PS/PSB 3 is shown in Fig. 5b. As can be seen, the reflection peak wavelength blue shifts with increasing angle of incidence. The color obtained with different viewing angles also confirms this feature. This is a typical characteristics of PC. Moreover, these results confirm that the NSP support photonic bandgap. The spectral characteristics of the samples changed with increasing concentration of SB comonomer, which caused a moderate change in the aspect ratio of protruding NSP $(<1.2)$. It may be noted that by introducing protrusion in the particle it is possible to tune the bandgap which is unusual from conventional microspheres. Table 2 shows how the concentration of comonomer SB influenced the particle size and morphology and the concomitant change in color of the self-assembled surface.

Due to the "anti-polyelectrolyte" behavior of $\mathrm{PSB},{ }^{38,39}$ these monodisperse NSPs behave as a truly salt-responsive PC array. Iridescence of PS/PSB particles could be tuned as a function of salt. Specifically, the low molecular weight electrolyte salt to screens the surface charges and promotes osmotic swelling
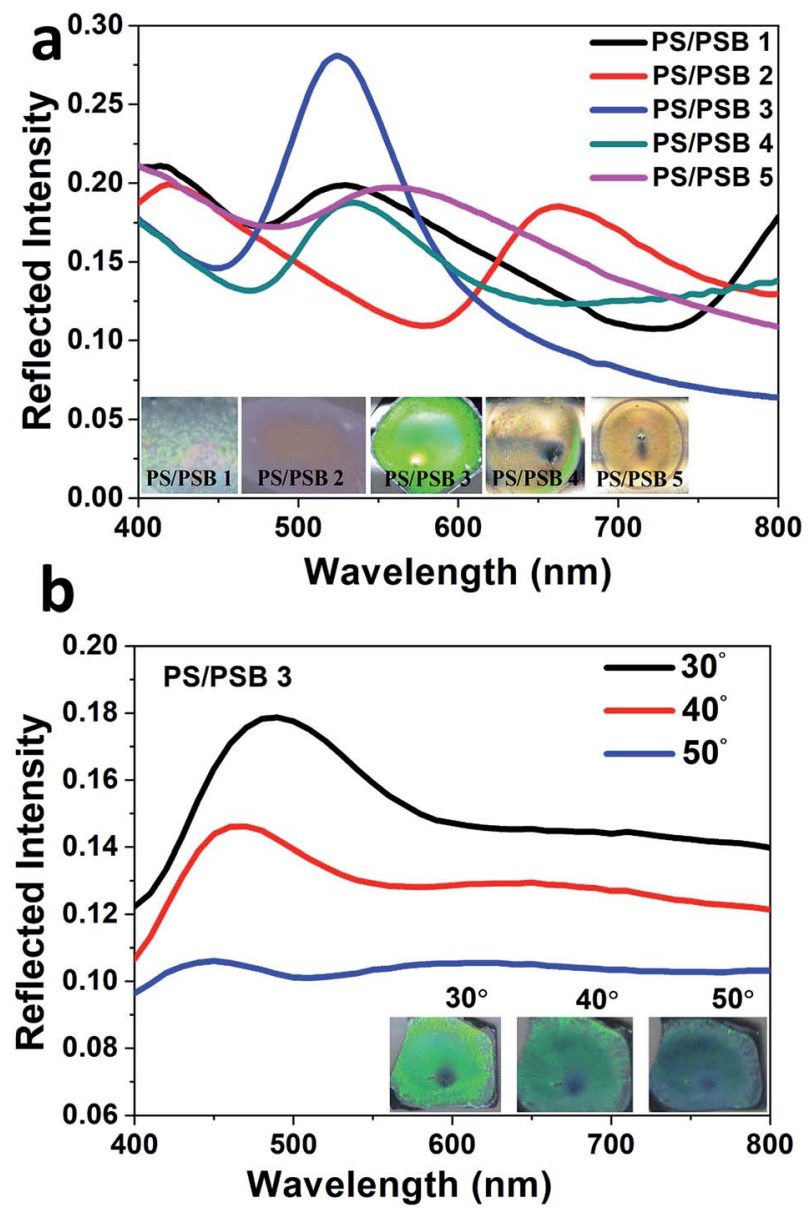

Fig. 5 (a) The reflection spectra of PS/PSB films at normal incidence (inset: vivid structural colors of NSPs on the silicon wafer at room temperature). (b) The angular reflection of PS/PSB 3 at $30^{\circ}, 40^{\circ}$ and $50^{\circ}$ respectively (inset: photographs of PS/PSB 3 at different angle). 
Table 2 Characteristics of PS/PSB polymer opal films

\begin{tabular}{llllll}
\hline Entry & Polymer code & SB $(\% \mathrm{w} / \mathrm{w})$ & Morphology & ${ }^{a} D$, SEM $(\mathrm{nm})$ & Structural color \\
\hline 1 & PS/PSB 1 & 1.5 & Sphere & $367 \pm 18$ & Blue-green \\
2 & PS/PSB 2 & 3.1 & Quasi sphere & $298 \pm 10$ & Purple-red \\
3 & PS/PSB 3 & 6.1 & Bulge & $207 \pm 9$ & Green \\
4 & PS/PSB 4 & 8.6 & Eye ball & $237 \pm 12$ & Yellow \\
5 & PS/PSB 5 & 9.9 & Snowman & $251 \pm 14$ & Yellow-orange
\end{tabular}

${ }^{a}$ Average particle diameter obtained from SEM; data represent the mean value and standard deviation based on the analysis of 75-100 particles.

through surface hydration of zwitterion of PS/PSB. This could cause a change in dimension of the NSPs leading to the observed difference in color. The angular reflection spectra of PS/PSB 3 at different salt concentrations is shown in Fig. 6a. It is clear that the reflection peak wavelength shifts with salt concentration; as a result, the color of PS/PSB 3 changes along with change in salt concentration (Fig. 6b). The photonic bandgap of salt responsive
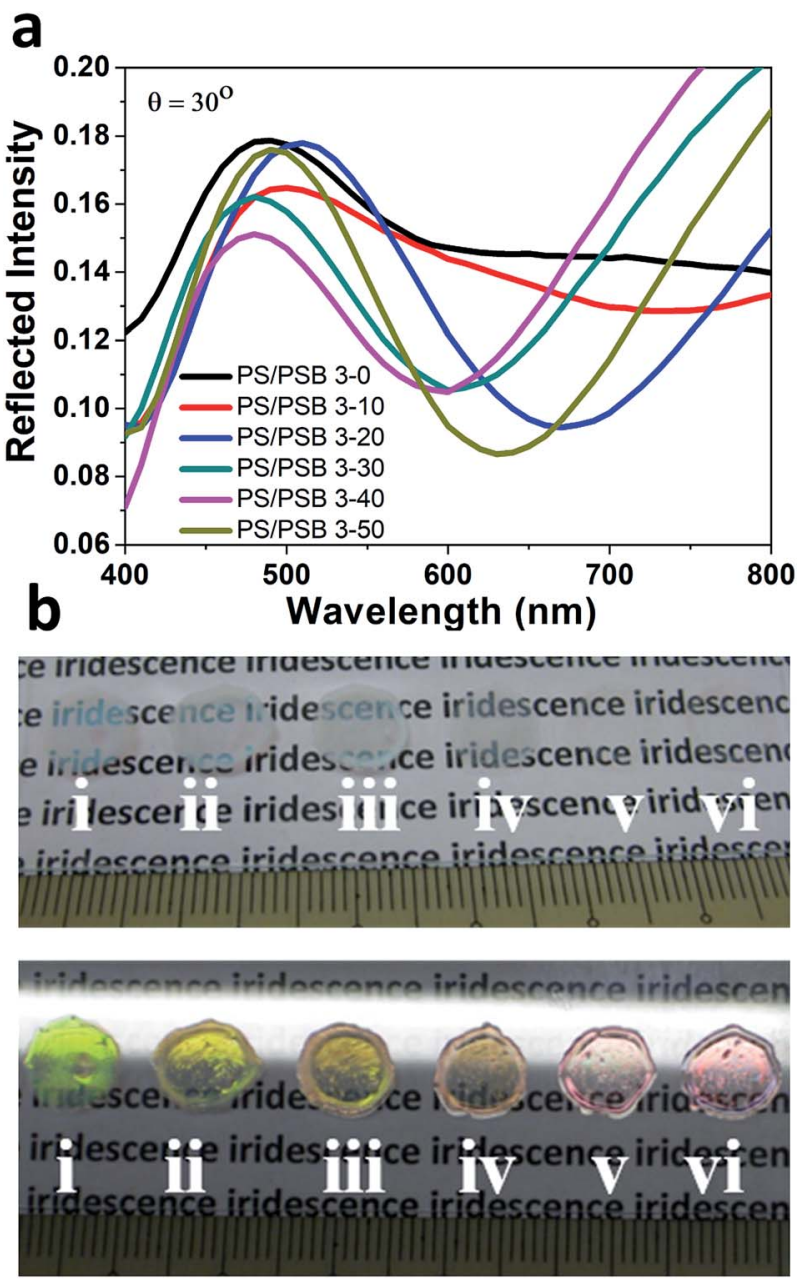

Fig. 6 (a) The angular reflection spectra of PS/PSB 3 in different salt concentrations (i) 0; (ii) 10; (iii) 20; (iv) 30; (v) 40; (vi) $50 \mu \mathrm{L}$ of $0.5 \mathrm{M} \mathrm{NaBr}$ in $100 \mu \mathrm{L}$ of PS/PSB 3 at $30^{\circ}$; (b) the corresponding photographs of tunable salt responsive opalescent of PS/PSB 3 (top: normal incidence showing no iridescence in the absence of light and bottom: angled reflection showing iridescence with the dynamic color change from green to red with salt solutions of varying in concentration in presence of light).
PC can be modulated across the visible spectrum by simply adjusting the salt concentration. The diverse structural colors could be tuned from green to yellow, yellow-orange, and red by changing the salt concentration, as shown in Fig. 6b. The phenomenon is similar to halo-solvatochromism. ${ }^{46}$ Notably, the transparency of films increases with increasing salt concentration (Fig. 6b(iv to vi)). At high salt concentration, due to the anisotropic nature of PS/PSB 3, light passes without being scattered unlike PS/PSB 3 at low salt concentrations which results in opaque film due to the scattering of light (Fig. 6b(i-iii)). The fabricated functional PC with different colors using a single system is unique as compared to those systems which change color by changing the size of the microsphere or other characteristics to obtain PC. ${ }^{\mathbf{1 7}, \mathbf{4 7}}$ The present sensing motif is solely based on saltphilic (halophilic) nature of PC which can possibly be extended to other environmental stimuli.

\section{Conclusions}

Here we presented for the first time a convenient way of preparing non-spherical PS/PSB particles that spontaneously self-assemble into functional PC arrays exhibiting full photonic bandgap in the visible region. This versatile synthetic approach of one-pot emulsifier-free emulsion polymerization that produces vivid iridescent tunable structural colors is amenable for scale-up. The SB comonomer was utilized to phase separate in the course of polymerization to achieve anisotropic NSP with controlled morphologies such as sphere, quasi-sphere, and protruded structures like bulge, eye-ball, and snowman-like nanostructures. The SB comonomer approach not only allows to tune the morphologies but also imparts stimuli-responsive characteristics. The functional NSP enables spontaneous self-assembly that reflects light without the use of any additive or template via a simple bottom-up approach. The functional NSP colloidal system can assemble into various unique packing arrangements. The demonstrated tunable structural colors cannot be found in particles formed by other conventional techniques. Moreover, the single system with functional flexibility enables a new direction for making stimuliresponsive photonic materials.

\section{Experimental}

\section{Materials and methods}

Styrene (Sty, 99\%, Sigma) was passed through a column filled with basic alumina to remove inhibitors and then stored at $-20{ }^{\circ} \mathrm{C}$ before use. The zwitterion sulfobetaine monomer (SB) 
was synthesized according to our previously reported procedure using $N$-(4-vinyl benzyl)- $N, N$-dimethylamine (90\%) which was purchased from ACROS and 1,3-propane sultone (99\%, Sigma) in dichloromethane at $50{ }^{\circ} \mathrm{C}$ for $24 \mathrm{~h} .{ }^{38}$ The initiator potassium persulfate $\left(\mathrm{K}_{2} \mathrm{~S}_{2} \mathrm{O}_{8}\right.$, KPS, 99.9\%, Sigma) was used without further purification. Other solvents and reagents were of analytical grade and purchased from Merck, VWR and Fisher Scientific. The dialysis tubing (Spectra/Por®) regenerated cellulose membrane with a molecular weight cutoff (MWCO) of $12 \mathrm{k}-14 \mathrm{k}$ Da were purchased from Spectrum Laboratories. Deionized water (DI water) was purified using a Millipore water purification system and filtered by $0.45 \mu \mathrm{m}$ filter before use. The silicon wafers and the glass substrate were supplied by Latech, Singapore. The silicon wafer and the glass substrate were cleaned using hexane, soap-solution and ethanol followed by drying under nitrogen flow and then kept in the oven at $50{ }^{\circ} \mathrm{C}$ before use.

General procedure for emulsifier-free aqueous emulsion polymerization of styrene using zwitterionic sulfobetaine (SB) comonomer (Table 1; entry 2; PS/PSB 2)

The polymerization was conducted in a dried three-neck flask (125 mL) equipped with a Premex magnetic coupling at the center of the neck and an overhead stirrer with 4-morton indents and 3-bladed impeller $\left(45^{\circ}\right)$. The reactor was heated by immersing in an oil bath and was maintained under nitrogen atmosphere throughout polymerization.

The colloidal PS/PSBs were synthesized by emulsifier-free aqueous emulsion polymerization of styrene (Sty) and zwitterionic sulfobetaine (SB) monomer. A typical polymerization procedure is described as follows. $4.54 \mathrm{~g}$ of styrene and $49 \mathrm{~mL}$ of DI water were first charged into the reactor at room temperature under nitrogen flow. Then the mixture was stirred at $350 \mathrm{rpm}$. Subsequently, $38 \mathrm{mg}$ of KPS dissolved in $1 \mathrm{~g}$ of DI water was added into the mixture. The mixture was purged with nitrogen. Then, the mixture was heated at $70{ }^{\circ} \mathrm{C}$ to initiate polymerization. It turned milky-white after $30 \mathrm{~min}$. The formation of polystyrene seed of 160-180 nm was confirmed by DLS. Simultaneously, $0.144 \mathrm{~g}$ of SB monomer was dissolved in DI water $(5 \mathrm{~g})$ and purged with nitrogen in a glass vial. The SB monomer solution was then added to the polymerizing emulsion dropwise for a period of $30 \mathrm{~min}$. The polymerization was continued for $24 \mathrm{~h}$. At different time intervals, about $0.5 \mathrm{~mL}$ of reaction mixture was withdrawn to determine the size and conversion. This was then diluted by DI water and subsequently precipitated in methanol. The particle size and the final conversion were determined using DLS and gravimetric method respectively. Finally, the polymer latex was cooled to room temperature. The dispersion was free of coagulum. The dispersion was directly transferred to the dialysis tubing and dialyzed against DI water (dialysis membrane, MWCO $=12 \mathrm{k}-14 \mathrm{k}$ ) for three days with DI water being replaced regularly to remove the residual unreacted monomers. Since the dispersion is free from surfactant, the dispersion is used as such for further studies. Conversion $=84.2 \%$; solid content $=6.3 \pm$ $0.16 \%$; SEC (RI detector) $M_{\mathrm{n}, \mathrm{SEC}}=185 \mathrm{k} \mathrm{g} \mathrm{mol}^{-1} ; M_{\mathrm{w}} / M_{\mathrm{n}}=2.81$.

A similar protocol was used in attempts to prepare zwitterion functionalized PS/PSB with different zwitterion sulfobetaine content of $1.5 \%, 3.1 \%, 6.1 \%$, and $9.9 \% \mathrm{w} / \mathrm{w}$ with respect to styrene. The composition of reactants is given in Table 1 . The resulting functionalized latex after dialysis was stored in a glass bottle for further characterization. The conversion, molecular weight, size, and polydispersity index (PDI) of functionalized PS/PSB prepared by emulsion polymerization are summarized in Table 1 and $\mathbf{S 1 . \dagger}$

\section{Fabrication of PS/PSB photonic crystal arrays}

The colloidal PC arrays were prepared by horizontal and vertical deposition on silica substrate. ${ }^{\mathbf{4 8 , 4 9}}$ For horizontal deposition, the substrate was wetted by drop casting the suspension on the surface with the as prepared concentration of PS/PSB without any dilution. The suspension was spread to cover the surface of the substrate fully. Then, the substrate was kept tilted until drying completely at room temperature. In the vertical deposition, the glass substrate was immersed in a PS/PSB dispersion and then dried at $50{ }^{\circ} \mathrm{C}$ for $24 \mathrm{~h}$ to obtain close packed PC array. PC array of PS/PSB self-assembled on the substrate upon evaporation of water under ambient conditions by capillary and electrostatic repulsive force.

\section{Characterization methods}

\section{Instrumentation}

The dried polymer particles were prepared by centrifugation and then dried in a vacuum oven at room temperature. The dried polymer particles were used for GPC, XPS, FTIR, and TGA analysis. The molecular weights were determined by size exclusion chromatography using a Viscotek TDA max with TDA 302 triple Detector Array by OmniSEC software. A size exclusion column combination of $2 \times$ PL Gel $10 \mu \mathrm{m}$ Mixed (500 to 10000000 ) was used in sequence for separation. The measurement was performed using filtered and sonicated HPLC grade THF as the eluent at $1.0 \mathrm{~mL} \mathrm{m^{-1 }}$ at $40{ }^{\circ} \mathrm{C}$. The columns were calibrated against commercially available polystyrene standard. Infrared spectra were recorded on a PerkinElmer Frontier NIR spectrometer by compressing the dried polymer with $\mathrm{KBr}$ into tablet. The sample measurements were performed from 400 to $4000 \mathrm{~cm}^{-1}$ with 64 scans per spectrum with the resolution of $4 \mathrm{~cm}^{-1}$. X-ray photoelectron spectroscopy (XPS) was obtained using ESCALAB 250, Thermo VG Scientific with a monochromatic Al K $\alpha$ X-ray source. The binding energies were referred to the $\mathrm{C} 1 \mathrm{~s}$ carbon peak at $284.6 \mathrm{eV}$. Thermogravimetric analysis (TGA) was performed on a TA instrument Q600 simultaneous TGA/DTA/DSC, model Q600/Nicolet 6700 under the nitrogen atmosphere. All samples were dried under vacuum at room temperature for $24 \mathrm{~h}$ prior to the analysis. In order to remove trace amounts of moisture, the samples were heated to $110{ }^{\circ} \mathrm{C}$ followed by an isothermal step for 30 minutes. The heating was set at the rate of $10{ }^{\circ} \mathrm{C}$ per min for temperature between 30 and $600{ }^{\circ} \mathrm{C}$.

\section{Scanning electron microscopy (SEM) and transmission electron microscopy (TEM)}

SEM measurement was employed to observe the size and the morphology of the polymer particles. SEM measurement was 
conducted by using a JEOL electron microscopy. The as prepared latex dispersion was placed on the glass substrate. After drying at room temperature for $24 \mathrm{~h}$. The samples were sputter coated with gold and then examined by SEM at an acceleration voltage of $2 \mathrm{kV}$. Colloidal PC of PS/PSBs were prepared by depositing aqueous dispersion on a silica substrate followed by drying at room temperature as described above.

For TEM analysis, a JEM-2100 high-resolution transmission electron microscope supplied by JEOL (Tokyo, Japan) was used. The colloidal polymer dispersion $(10 \mu \mathrm{L})$ was diluted using DI water $(1000 \mu \mathrm{L})$. Then aliquot containing particles was added dropwise onto carbon-coated copper grid and allowed to dry for $24 \mathrm{~h}$.

\section{Cryo-transmission electron microscopy (Cryo-TEM)}

Vitrification of the latex particle samples was carried out using Vitrobot (FEI Vitrobot Mark IV, Hillsboro, OR, USA). The vitrification condition for all samples was as the following: room temperature, $100 \%$ humidity, blot time of $2 \mathrm{~s}$ and blot force of 1 . The sample (5 $\mu \mathrm{L}$ at 1-2 wt\%) was applied onto a grid (Quantifoil, R2/2, Holey carbon film) which was freshly glowdischarged prior to use at $20 \mathrm{~mA}$ for $60 \mathrm{~s}$. Excess of sample was blotted away with filter paper to leave a thin film on the grid before being vitrified in liquid ethane.

Cryo-TEM imaging was performed on FEI Titan Krios equipped with an automated sample loader, and a Field Emission Gun (FEG) operating at $300 \mathrm{kV}$. Images were recorded with Falcon II camera $(4 \times 4)$ with the magnification of 29000 .

\section{Dynamic light scattering (DLS) and aqueous electrophoresis}

The average hydrodynamic diameter and polydispersity index (PDI) of PS/PSB colloids were evaluated by a Zetasizer Nano ZS dynamic light scattering (DLS) instrument (Malvern, UK) with a Peltier heating system. The wavelength of $633 \mathrm{~nm}$ and the scattering angle of $173^{\circ}$ were fixed at room temperature. The zeta potential of each particle was obtained from the electrophoresis mobility measured using the same Malvern Zetasizer NanoZS using disposable capillary cells in the presence of $1 \mathrm{mM}$ $\mathrm{NaCl}$ solution. The zeta potential was performed five times with $0.001 \% \mathrm{v} / \mathrm{v}$ aqueous latex and each measurement was repeated 5 times. Prior to analysis, DLS and zeta potential were calibrated using polystyrene latex standard, product number LTX3060A and zeta potential transfer standard using DTS 1235.

\section{Reflection measurements}

A UV/Visible microspectrophotometer (JACSO, MSV-5200) with a wavelength spectroscopic resolution of $2 \mathrm{~nm}$ was used to measure the reflection spectra at normal incidence. The beam diameter was set to $50 \mu \mathrm{m}$. The angular reflectance spectra were acquired using a variable angle spectroscopic ellipsometry (J. A. Woollam Co., Inc, V-VASE) with a wavelength spectroscopic resolution of $2 \mathrm{~nm}$.

\section{Conflicts of interest}

There are no conflict of interest to declare.

\section{Acknowledgements}

This project was funded by the Agency for Science, Technology and Research (A*STAR), Singapore under Advanced Surfaces Programme (Project title: Light responsive polymers; Grant no. 1523700105). The authors thank Ms Melissa Prawirasatya for TEM analysis. XPS, EA, SEM, FTIR, and TGA was conducted at the Institute of Chemical Engineering Sciences, Central Lab Facility.

\section{Notes and references}

1 P. A. P. A. Lovell and M. S. El-Aasser, Emulsion polymerization and emulsion polymers, J. Wiley, 1997.

2 A. Imhof, in Nanoscale Materials, ed. L. M. Liz-Marzán and P. V. Kamat, Springer, USA, Boston, MA, 2003, pp. 423-454.

3 Y. Xia, B. Gates, Y. Yin and Y. Lu, Adv. Mater., 2000, 12, 693713.

4 A. D. Dinsmore, J. C. Crocker and A. G. Yodh, Curr. Opin. Colloid Interface Sci., 1998, 3, 5-11.

5 B. Li, D. Zhou and Y. Han, Nat. Rev. Mater., 2016, 1, 15011. 6 R. van Dommelen, P. Fanzio and L. Sasso, Adv. Colloid Interface Sci., 2018, 251, 97-114.

7 J.-G. Park, S.-H. Kim, S. Magkiriadou, T. M. Choi, Y.-S. Kim and V. N. Manoharan, Angew. Chem., Int. Ed., 2014, 53, 2899-2903.

8 F. Fu, L. Shang, F. Zheng, Z. Chen, H. Wang, J. Wang, Z. Gu and Y. Zhao, ACS Appl. Mater. Interfaces, 2016, 8, 1384013848.

9 G. Iasilli, R. Francischello, P. Lova, S. Silvano, A. Surace, G. Pesce, M. Alloisio, M. Patrini, M. Shimizu, D. Comoretto and A. Pucci, Mater. Chem. Front., 2019, 3, 429-436.

10 E. Armstrong and C. O'Dwyer, J. Mater. Chem. C, 2015, 3, 6109-6143.

11 J. F. Galisteo-López, M. Ibisate, R. Sapienza, L. S. FroufePérez, Á. Blanco and C. López, Adv. Mater., 2011, 23, 30-69.

12 C. E. Finlayson and J. J. Baumberg, Polym. Int., 2013, 62, 1403-1407.

13 Y. Yue and J. P. Gong, J. Photochem. Photobiol., C, 2015, 23, 45-67.

14 H. Zheng and S. Ravaine, Crystals, 2016, 6, 54.

15 J. Hou, M. Li and Y. Song, Nano Today, 2018, 22, 132-144.

16 J. Hou, M. Li and Y. Song, Angew. Chem., Int. Ed., 2018, 57, 2544-2553.

17 L. Nucara, V. Piazza, F. Greco, V. Robbiano, V. Cappello, M. Gemmi, F. Cacialli and V. Mattoli, ACS Appl. Mater. Interfaces, 2017, 9, 4818-4827.

18 S.-H. Kim, S. Y. Lee, S.-M. Yang and G.-R. Yi, NPG Asia Mater., 2011, 3, 25.

19 Y. Zhao, L. Shang, Y. Cheng and Z. Gu, Acc. Chem. Res., 2014, 47, 3632-3642.

20 Y. Zhang, J. Wang, Y. Huang, Y. Song and L. Jiang, J. Mater. Chem., 2011, 21, 14113-14126.

21 G. von Freymann, V. Kitaev, B. V. Lotsch and G. A. Ozin, Chem. Soc. Rev., 2013, 42, 2528-2554.

22 W. L. Miller, B. Bozorgui and A. Cacciuto, J. Chem. Phys., 2010, 132, 134901. 
23 C. G. Schaefer, M. Gallei, J. T. Zahn, J. Engelhardt, G. P. Hellmann and M. Rehahn, Chem. Mater., 2013, 25, 2309-2318.

24 S. C. Glotzer and M. J. Solomon, Nat. Mater., 2007, 6, 557.

25 X. Bouju, É. Duguet, F. Gauffre, C. R. Henry, M. L. Kahn, P. Mélinon and S. Ravaine, Adv. Mater., 2018, 30, 1706558.

26 J. D. Forster, J.-G. Park, M. Mittal, H. Noh, C. F. Schreck, C. S. O'Hern, H. Cao, E. M. Furst and E. R. Dufresne, ACS Nano, 2011, 5, 6695-6700.

27 V. Lotito and T. Zambelli, Adv. Colloid Interface Sci., 2017, 246, 217-274.

28 J.-W. Kim, R. J. Larsen and D. A. Weitz, J. Am. Chem. Soc., 2006, 128, 14374-14377.

29 S. Sacanna and D. J. Pine, Curr. Opin. Colloid Interface Sci., 2011, 16, 96-105.

30 B. Yu, H. Cong, Q. Peng, C. Gu, Q. Tang, X. Xu, C. Tian and F. Zhai, Adv. Colloid Interface Sci., 2018, 256, 126-151.

31 C. E. Reese and S. A. Asher, J. Colloid Interface Sci., 2002, 248, 41-46.

32 D. C. Sundberg and Y. G. Durant, Polym. React. Eng., 2003, 11, 379-432.

33 J. W. Kim and K. D. Suh, J. Ind. Eng. Chem., 2008, 14, 1-9.

34 H. R. Sheu, M. S. El-Aasser and J. W. Vanderhoff, J. Polym. Sci., Part A: Polym. Chem., 1990, 28, 629-651.

35 N. J. Warren and S. P. Armes, J. Am. Chem. Soc., 2014, 136, 10174-10185.
36 K. E. B. Doncom, N. J. Warren and S. P. Armes, Polym. Chem., 2015, 6, 7264-7273.

37 V. A. Vasantha, S. Jana, A. Parthiban and J. G. Vancso, $R S C$ Adv., 2014, 4, 22596-22600.

38 V. A. Vasantha, C. Junhui, Z. Wenguang, A. M. van Herk and A. Parthiban, Langmuir, 2019, 35, 1465-1474.

39 V. Arjunan Vasantha, A. Oh Biying and A. Parthiban, J. Appl. Polym. Sci., 2018, 135, 46178.

40 K. J. Lee, J. Yoon and J. Lahann, Curr. Opin. Colloid Interface Sci., 2011, 16, 195-202.

41 E. B. Mock, H. De Bruyn, B. S. Hawkett, R. G. Gilbert and C. F. Zukoski, Langmuir, 2006, 22, 4037-4043.

42 B. Charleux, G. Delaittre, J. Rieger and F. D'Agosto, Macromolecules, 2012, 45, 6753-6765.

43 L. Wu, J. Jasinski and S. Krishnan, J. Appl. Polym. Sci., 2012, 124, 2154-2170.

44 V. Arjunan Vasantha, C. Junhui, T. B. Ying and A. Parthiban, Langmuir, 2015, 31, 11124-11134.

45 H. P. Blom, M. Gauthier, K. Li and K. E. Nielsen, J. Appl. Polym. Sci., 1998, 70, 227-236.

46 C. Reichardt, Chem. Soc. Rev., 1992, 21, 147-153.

47 K. Lee and S. A. Asher, J. Am. Chem. Soc., 2000, 122, 95349537.

48 A. S. Dimitrov and K. Nagayama, Langmuir, 1996, 12, 13031311.

49 Q. Yan, Z. Zhou and X. S. Zhao, Langmuir, 2005, 21, 31583164. 